\title{
Self Adaptive Coalitions in Multiagent Systems
}

\author{
Guido Boella \\ University of Turin, Italy \\ guido@di.unito.it
}

\author{
Leendert van der Torre \\ University of Luxembourg, Luxembourg \\ leendert@vandertorre.com
}

\author{
Serena Villata \\ University of Turin, Italy \\ villata@di.unito.it
}

\begin{abstract}
Coalitions are usually defined with respect to a static framework of dependencies among agents. In this paper we propose a dynamic view of dependence networks to enable dynamic coalitions which can self adapt to a situation by exploiting the possibility to trigger other agents' goals.
\end{abstract}

Social viewpoints become more popular in multiagent systems (MAS), since the representation of MAS as, for example, social networks, dependence networks, organizations, or normative systems, focusses on the interactions among the agents and facilitates the development of interaction mechanisms, agreement technologies or adaptive organizations. We [1] provide a semi-formal definition of social viewpoints on MAS, and relate views of these viewpoints to each other using abstraction and refinement relations. For example, a detailed BDI model can be abstracted to a dependence network as used in Tropos [4] where they show how to use these formal representations to define criteria for coalition formation [2], or measures for MAS inspired by social network analysis (SNA) [3]. However, they consider only absolute goals and static dependence networks. This prevents modelling more adaptive situations, where for example a coalition dynamically self adapts to a situation: a member of the coalition triggers a goal of another agent to make it dependent on the coalition so that the agent has an advantage in entering the coalition and give its contribution in turn.

In this paper we therefore address the following three problems to introduce self adaptability in our work on social viewpoints on MAS: How to define a dynamic dependence network for agents with conditional goals? Which kind of powers can be distinguished in a dynamic dependence network? How to define self adaptive coalitions for dynamic dependence networks?

Like in our previous work [1], we use the simplest representation possible to relate the views. For example, we do not consider actions, tasks, planning, or resources.

In planning, goals are given. Therefore, many models define the goals of a set of agents $A$ by a function goals :
$A \rightarrow 2^{G}$, where $G$ is the complete set of goals. However, in many agent programming languages and architectures, goals are conditional and can be generated. We therefore extend the mind view of [1] with conditional goals.

Definition 1 (Mind view) is represented by the tuple $\left\langle A, G, X\right.$, goals $: A \times 2^{X} \rightarrow 2^{G}$, skills $\left.: A \rightarrow 2^{X}, R: 2^{X} \rightarrow 2^{G}\right\rangle$ where $A$ is a set of agents, $G$ is a set of goals, $X$ is a set of decisions and $R$ is a set of rules.

Example 1 (See Figure) $A=\left\{a_{1}, a_{2}, a_{3}, a_{4}, a_{5}\right\}$ and $G=\left\{g_{1}, g_{2}, g_{3}, g_{4}\right\}$ and $X=\left\{x_{1}, x_{2}, x_{3}, x_{4}, x_{5}\right\}$;

goals $\left(a_{1}, \emptyset\right)=\left\{g_{1}\right\}, \quad$ goals $\left(a_{3}, \emptyset\right)=\left\{g_{2}\right\}$, goals $\left(a_{5},\left\{x_{1}, x_{3}\right\}\right)=\left\{g_{3}\right\}, \quad$ goals $\left(a_{5},\left\{x_{5}\right\}\right)=\left\{g_{3}\right\}$, goals $\left(a_{2},\left\{x_{1}\right\}\right)=\left\{g_{4}\right\}$;

skills $\left(a_{1}\right)=\left\{x_{1}\right\}$, skills $\left(a_{2}\right)=\left\{x_{2}\right\}$, skills $\left(a_{3}\right)=\left\{x_{1}, x_{3}\right\}$, $\operatorname{skills}\left(a_{4}\right)=\left\{x_{5}\right\}, \operatorname{skills}\left(a_{5}\right)=\left\{x_{4}\right\}$;

$R\left(\left\{x_{4}\right\}\right)=\left\{g_{1}, g_{2}\right\}, R\left(\left\{x_{2}\right\}\right)=\left\{g_{3}\right\}, R\left(\left\{x_{1}, x_{3}\right\}\right)=\left\{g_{4}\right\} ;$

With conditional goals, two kinds of powers can be distinguished: to trigger a goal, and to fulfill it.

Definition 2 (Power view) is represented by the tuple $\left\langle A, G\right.$, power-goals $: 2^{A} \rightarrow 2^{(A \times G)}$, power $\left.: 2^{A} \rightarrow 2^{G}\right\rangle$

A set of agents $B$ has the power to see to it that agent $a$ has the goal $g$, written as $(a, g) \in$ power-goals $(B)$, if and only if there are a set of decisions of $B$ such that $g$ becomes a goal of $a$. A set of agents $B$ has the power to see to goal $g$ if and only if there are a set of decisions of $B$ such that $g$ is a consequence of it.

Definition $3\langle A, G$, power-goals, power $\rangle$ is an abstraction from $\langle A, G, X$, goals, skills, $R\rangle$ if and only if:

- $(a, g) \in$ power-goals $(B)$ if and only if goals $(B)=$ $\cup\{$ skills $(b) \mid b \in B\}$ such that $g \in$ goals $(a, Y)$, and

- $g \in \operatorname{power}(B)$ if and only if $\exists Y \subseteq$ skills $(B)$ such that $g \in R(Y)$.

Example $2 \quad A$ and $G$; power-goals $(\emptyset)=$ $\left\{\left(a_{1}, g_{1}\right),\left(a_{3}, g_{2}\right)\right\}, \quad$ power-goals $\left(\left\{a_{3}\right\}\right)=\left\{\left(a_{5}, g_{3}\right)\right\}$, power-goals $\left(\left\{a_{4}\right\}\right)=\left\{\left(a_{5}, g_{3}\right)\right\}$, power-goals $\left(\left\{a_{1}\right\}\right)=$ $\left\{\left(a_{2}, g_{4}\right)\right\}$; power $\left(\left\{a_{5}\right\}\right)=\left\{g_{1}, g_{2}\right\}$, power $\left(\left\{a_{3}\right\}\right)=\left\{g_{4}\right\}$, power $\left(\left\{a_{2}\right\}\right)=\left\{g_{3}\right\}$; 


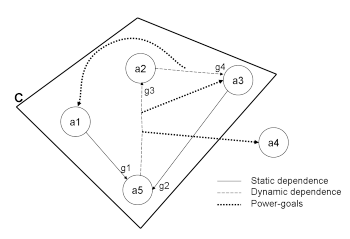

Due to the power to create goals, dependence relations are no longer static but can be created by the agents. We therefore have to extend the dependence networks developed by Conte and Sichman [6] to dynamic ones as in [5].

Definition 4 (Dynamic dependence view) A dynamic dependence network $(D D N)$ is a tuple $\langle A, G$, dyndep $\rangle$ where $A$ and $G$ are as before, and dyndep : $A \times 2^{A} \times 2^{A} \rightarrow 2^{2^{G}}$ is a function that relates with each triple of a agent and two sets of agents all the sets of goals on which the first depends on the second, if the third creates the dependency.

Abstracting power view to a dynamic dependence network can be done as below. Here, the creation of a dynamic dependency is based only on the power to create goals. In other models, creating a dependency can also be due to creation of new skills of an agent.

Definition $5\langle A, G$, dyndep $\rangle \quad$ is an abstraction of $\langle A, G$,power-goals, power $\rangle$, if we have $H \in$ dyndep $(a, B, C)$ iff 1) $\forall g \in H:(a, g) \in$ power-goals $(C)$, and 2) $H \subseteq$ power $(B)$.

Example 3 A and $G$; dyndep $\left(a_{1},\left\{a_{5}\right\}, \emptyset\right)=\left\{\left\{g_{1}\right\}\right\}$, dyndep $\left(a_{3},\left\{a_{5}\right\}, \emptyset\right)=\left\{\left\{g_{2}\right\}\right\}$, dyndep $\left(a_{2},\left\{a_{3}\right\},\left\{a_{1}\right\}\right)=$ $\left\{\left\{g_{4}\right\}\right\}, \quad$ dyndep $\left(a_{5},\left\{a_{2}\right\},\left\{a_{3}\right\}\right)=\left\{\left\{g_{3}\right\}\right\}$, dyndep $\left(a_{5},\left\{a_{2}\right\},\left\{a_{4}\right\}\right)=\left\{\left\{g_{3}\right\}\right\}$;

We combine these two abstractions, abstracting mind view to a dynamic dependence network as follows.

Proposition $1\langle A, G$, dyndep $\rangle$ is an abstraction of $\langle A, G, X$, goals, skills, $R\rangle$, if we have $H \in \operatorname{dyndep}(a, B, C)$ iff 1) $\exists Y \subseteq \operatorname{skills}(C)$ such that $H \subseteq \operatorname{goals}(a, Y)$, and 2) $\exists Y \subseteq \operatorname{skills}(B)$ such that $H \subseteq R(Y)$

Finally, we define reciprocity based coalitions for dynamic dependence networks. Like in [1], we represent the coalition not only by a set of agents, as in game theory, but as a set of agents together with a partial dynamic dependence relation. Intuitively, the dynamic dependence relation represents the "contract" of the coalition: if $H \in$ dyndep $(a, B, D)$, then the set of agents $D$ is committed to create the dependency, and the set of agents $B$ is committed to see to the goals $H$ of agent $a$. The rationality constraints on reciprocity based coalitions are that each agent contributes something, and receives something back.
Definition 6 (Reciprocity based Coalition) Given $a d y$ namic dependence network $\langle A, G$, dyndep $\rangle$, a reciprocity based coalition is represented by coalition $C \subseteq A$ together with dynamic dependencies dyndep $\subseteq$ dyndep, such that

- if $\exists b, B, D, H$ with $H \in$ dyndep $^{\prime}(a, B, D)$ then $a \in C$, $B \subseteq C$ and $D \subseteq C$ (the domain of dyndep' contains only agents in coalition $C$ ), and

- for each agent $a \in C$ we have $\exists b, B, D, H$ with $H \in$ dyndep $(b, B, D)$ such that $a \in B \cup D$ (agent a contributes either creating a dependency or fulfilling $a$ goal), and

- for each agent $a \in C \quad \exists B, D, H$ with $H \in$ dyndep' $(a, B, D)$ (agent a receives something from $C$ ).

Example 4 Coalition $C=\left\{a_{1}, a_{2}, a_{3}, a_{5}\right\}$. Agent $a_{4}$ is excluded from $C$ because it has a power-goal (the same as $a_{3}$ ) but he does not depend on any agent in $C$.

Summarizing, we define dynamic dependence networks by making the dependence relation conditional to the agents that have the power to create the relation. We distinguish two kinds of power, not only to fulfill goals as in static networks but also the power to create dependencies. Coalitions are defined by "contracts" in which each agent both contributes to and profit from it: the coalition can self adapt to the situation by exploiting - via goal triggering - the dynamics of DNs to exploit opportunities which are not currently present. Further researches are the use of our new theory for coalition formation, e.g., when two agents can make the other depend on itself and thus create a potential coalition? Do these new ways to create coalitions make the system more efficient? Moreover, new measures have to be defined for DDNs, finding inspiration in dynamic SNA.

\section{References}

[1] G. Boella, L. Sauro, and L. van der Torre. Social viewpoints on multiagent systems. In Procs of AAMAS'04, pages 1358 1359, 2004.

[2] G. Boella, L. Sauro, and L. van der Torre. Strengthening admissible coalitions. In Procs of ECAI'06, pages 195-199, 2006.

[3] G. Boella, L. Sauro, and L. van der Torre. From social power to social importance. Web Intelligence and Agent Systems, pages 393-404, 2007.

[4] P. Bresciani, A. Perini, P. Giorgini, F. Giunchiglia, and J. Mylopoulos. Tropos: An agent-oriented software development methodology. Journal of AAMAS, 8:203-236, 2004.

[5] P. Caire, S. Villata, L. van der Torre, and G. Boella. Conviviality masks in role-based institutions multi-agent teleconferencing in virtual worlds. In Procs of AAMAS'08, pages 1265-1269, 2008.

[6] J. S. Sichman and R. Conte. Multi-agent dependence by dependence graphs. In Procs of AAMAS'02, pages 483-490, 2002. 